\title{
Effects of Polymorphonuclear Leukocyte Transmigration on the Barrier Function of Cultured Intestinal Epithelial Monolayers
}

\author{
Shirin Nash, Joan Stafford, and James L. Madara \\ Departments of Pathology (Gastrointestinal Pathology), Brigham and Women's Hospital and Harvard Medical School, and the \\ Harvard Digestive Diseases Center, Boston, Massachusetts 02115
}

\begin{abstract}
We describe a model to study the effects of polymorphonuclear leukocyte (PMN) transmigration on the intestinal epithelial barrier. Human PMN were induced to transmigrate across high resistance monolayers of a cultured human intestinal epithelial cell line $\left(\mathbf{T}_{84}\right.$ cells) by chemotactic gradients produced by formyl methionyl leucyl phenylalanine (FMLP). With maximal transmigration monolayer resistance decreased by $48 \pm 12.6 \%$ in $15 \mathrm{~min}$ and by $83 \pm 1.6 \%$ in $60 \mathrm{~min}$. This response was dependent on the size of the FMLP gradient and the density of PMN transmigration. The decrease in resistance correlated with number of PMN migrating across monolayers, and was accompanied by increases in flux of paracellular tracers. Macromolecular tracer studies localized the leak sites to foci at which PMN impaled the epithelium. Removal of the chemotactic gradient led to restoration of baseline resistance within 18 h. PMN transmigration across intestinal epithelial monolayers occurs via intercellular occluding junctions and may be associated with a reversible increase in epithelial permeability.
\end{abstract}

\section{Introduction}

Migration of polymorphonuclear leukocytes (PMN) across intestinal epithelium occurs in a variety of idiopathic and infectious inflammatory diseases afflicting the small intestine and colon. This histologic finding, which may lead to the accumulation of neutrophilic exudate within dilated crypts (crypt abscesses), is utilized by pathologists to determine the degree of "activity" of the inflammatory process $(1,2)$. Mucosal preparations from patients with inflammatory bowel disease may show decreased epithelial barrier function, as assessed by resistance measurements, even in the absence of detectable ulceration (3). This decrease in epithelial barrier function in inflammatory diseases is probably due to the additive effects of many interwoven events. One factor that might contribute to intestinal epithelial barrier dysfunction is the impalement of the epithelium by transmigrating PMN. In these experiments, we have utilized electrophysiologic, morphologic, and tracer techniques to examine the effects of human PMN transmigration on the barrier function of monolayers composed of human

Address reprint requests to Dr. Nash, Department of Pathology, Brigham and Women's Hospital, 75 Francis Street, Boston, MA 02115. 1987.

Received for publication 4 March 1987 and in revised form 4 May

J. Clin. Invest.

(c) The American Society for Clinical Investigation, Inc.

$0021-9738 / 87 / 10 / 1104 / 10 \quad \$ 2.00$

Volume 80, October 1987, 1104-1113 intestinal epithelial cells ( $\mathrm{T}_{84}$ cells). We have previously shown that these monolayers exhibit stable, high resistance to passive ion flow and consist of confluent polarized columnar cells (4, 5). Our current studies show that in the presence of an optimal transepithelial chemotactic gradient, substantial PMN transmigration across this epithelium is accompanied by large, but reversible, barrier defects. In contrast, in the presence of only a small number of transmigrating PMN, the epithelium exhibits a remarkable ability to maintain barrier integrity. This system may serve as a useful model of intestinal inflammation, in which interactions between epithelial cells and inflammatory cells, such as PMN, can be better characterized.

\section{Methods}

Cell culture. $T_{84}$ cells were passaged and grown on $1.98 \mathrm{~cm}^{2}$ ring mounted, collagen coated, permeable supports after the method of Dharmsathaphorn as previously described (4-6). 200 monolayers composed of cells from passages 16-47 were used in these experiments. Transepithelial resistances developed by these monolayers varied between 200 and $2,400 \mathrm{ohms} \mathrm{cm}^{2}$ with the majority being $>500 \mathrm{ohms}$ $\mathrm{cm}^{2}$. We intentionally utilized monolayers with variable resistances, since others have suggested that the density of chemotactic migration of PMN across epithelial monolayers may be directly affected by the baseline resistance of the monolayer $(7,8)$. Since our experimental system allows us to record serial resistance readings from individual monolayers (see below), we were directly able to test this hypothesis.

We also utilized 12 monolayers of a high resistance Madin-Darby canine kidney (MDCK) ${ }^{1}$ cell line to determine whether the major findings of this study were restricted to $\mathrm{T}_{\mathbf{8 4}}$ intestinal monolayers, or were more broadly applicable. MDCK monolayers were grown in Dulbecco-Vogt modified Eagle's medium supplemented with $1 \%$ penicillin-streptomycin, $1 \%$ glutamine and $10 \%$ fetal calf serum, pH 7.4. Subcultures and monolayers were made as for $T_{84}$ cells. These monolayers developed resistances ranging from $536 \mathrm{ohms} \mathrm{cm}^{2}$ to 1,834 ohms $\mathrm{cm}^{2}$ with a mean of $1,128 \mathrm{ohms} \mathrm{cm}^{2}$.

$P M N$ isolation. PMN were routinely isolated from anticoagulated (sodium citrate $13.2 \mathrm{~g}$, dextrose $11.2 \mathrm{~g}$, in $500 \mathrm{ml}$ water, $\mathrm{pH}$ 6.5) whole blood collected by venipuncture from normal donors of both sexes (9). The PMN collection procedure was approved by the Brigham and Women's Hospital Human Subjects Committee (protocol 85-1465-1). Platelet-rich plasma was removed after centrifugation $(1,000 \mathrm{~g}, 3 \mathrm{~min})$ and the cell pellet diluted 1:3 with Hanks' balanced salt solution without $\mathrm{Ca}^{2+}$ and $\mathrm{Mg}^{2+}$ (M. A. Bioproducts, Walkersville, MD) at $37^{\circ} \mathrm{C}$. PMN were separated by centrifugation $(500 \mathrm{~g}, 40 \mathrm{~min}$ ) on lymphocyte separation medium (Litton Bionetics, Kensington, MD), followed by dextran sedimentation at $37^{\circ} \mathrm{C}$ and hypotonic lysis of contaminating red blood cells. PMN (95\% pure) with $98 \%$ viability by dye exclusion were suspended in tissue culture medium (described above) at $10^{7}$ $\mathrm{PMN} / \mathrm{ml}$ of fluid, held at $4^{\circ} \mathrm{C}$, and used for experiments within $1 \mathrm{~h}$ after isolation.

1. Abbreviations used in this paper: EM, electron micrograph; FMLP, $\mathrm{N}$-formyl methionyl leucyl phenylalanine; HRP, horseradish peroxidase; MDCK, Madin-Darby canine kidney. 
Induction of PMN transmigration. Ring-mounted monolayers were elevated on glass beads in plastic tissue-culture wells to allow mixing of solution under the ring-monolayer assembly. Monolayers were oriented such that the apical ("mucosal") aspect of the cells faced the upper compartment. PMN were suspended in a final volume of 1 $\mathrm{ml}$ and applied to the apical surface of monolayers and chemotactic gradients were established across monolayers by adding $N$-formylmethionyl-leucyl-phenylalaline (FMLP) (Sigma Chemical Co., St. Louis, MO) to the basal ("serosal") compartment. Incubation was carried out at $37^{\circ} \mathrm{C}$ in a $95 \%$ air $/ 5 \% \mathrm{CO}_{2}$ atmosphere.

To determine if the functional effects of PMN transmigration on epithelial barrier function were reversible, we removed the chemotactic gradient in a subset of experiments and allowed recovery to occur for varying time periods.

To determine if the effects of PMN transmigration on epithelial barrier function were independent of the direction of PMN movement we reversed the direction of the chemotactic gradient by adding FMLP $10^{-7} \mathrm{M}$ to the apical compartment of those monolayers that had been used for the resealing experiments. Resistance measurements were made after incubation of monolayers at $37^{\circ} \mathrm{C}$ for $1 \mathrm{~h}$.

Ussing chamber studies. Transepithelial resistance of $\mathrm{T}_{84}$ monolayers was determined in low turbulence Ussing chambers, modified to accept ring-mounted monolayers as previously described (4-6). Each reservoir was connected via agar bridges to voltage sensitive calomel electrodes and to $\mathrm{Ag}-\mathrm{AgCl}$ current passing electrodes. Voltage deflections elicited by the passage of $100 \mu \mathrm{A}$ current pulses were used to determine transepithelial resistance. We have previously determined that the current voltage relationship is linear up to the $100 \mu \mathrm{A}$ range (data not shown). After equilibration, resistance was measured and recorded as the absolute resistance minus the fluid resistance. The resistance of the collagen-coated filter (Nuclepore Corp., Pleasanton, CA) $\left(<3 \mathrm{ohms} \mathrm{cm}^{2}\right.$ ) was neglected. In a set of control $\mathrm{T}_{84}$ monolayers, we determined that we could serially remove and reinsert monolayers into Ussing chambers without affecting baseline resistance (see $\mathrm{Re}$ sults). We were thus able to take initial and post experimental resistance readings on the same monolayer. Other data (see Results) verified that experimentally modified barrier function does not change for at least $\mathbf{4 h}$ after removal of experimental conditions. Therefore, it was appropriate to obtain functional data (resistance response) on experimental tissues by such Ussing chamber studies.

Transepithelial flux rates of mannitol and inulin were carried out on $\mathrm{T}_{84}$ monolayers under experimental conditions and compared with flux rates obtained from control monolayers of comparable baseline resistance as previously described (4).

Morphologic techniques. At the end of experiments, portions of monolayers were processed for thick and thin sections and for freeze fracture as previously described $(4,5)$. Half of most monolayers was utilized to quantitate PMN transmigration (chemotaxis assay, see below).

Horseradish peroxidase (HRP), (Sigma type 2; 40,000 molecular weight), was used to assess the permeability of occluding junctions to macromolecules. HRP $0.5 \%$ in tissue culture fluid was applied to the apical aspect of experimental and control monolayers for the last 20 min of hour-long chemotaxis experiments. Control monolayers had either no FMLP in the basal compartment with PMN on the apical side or, alternatively, no FMLP in the basal compartment and also no PMN in the apical compartment. Tissues were removed from the chemotaxis chambers, fixed and processed for peroxidase localization (10) and electron microscopy.

Chemotaxis assay. We took advantage of the presence of endogenous peroxidase in PMN but not in $\mathrm{T}_{84}$ cells and stained portions of monolayers for peroxidase localization by the method of Graham and Karnovsky (10). Monolayers were then dehydrated through graded alcohols and mounted as flat sheets on glass slides for quantitation of transmigrated PMN. Using the light microscope, the surface of each monolayer was identified by the presence of rare contaminating red blood cells that also contain endogenous peroxidase and are uniformly stained by the above method. The base of the monolayer was identified by locating the pores in the Nuclepore filter. PMN between these two surfaces (i.e., subjunctional PMN) exhibited granular cytoplasmic staining and were counted and expressed as the chemotactic response in $\mathrm{PMN} / \mathrm{cm}^{2}$ of monolayer. 1- $\mu \mathrm{m}$ sections confirmed that PMN counted by this method were located between $T_{84}$ cells and the underlying collagen-coated filter and represented PMN that had migrated through $\mathrm{T}_{84}$ cell monolayers.

We excluded the possibility that significant numbers of transmigrating PMN may migrate through Nuclepore filters into the serosal compartment by performing PMN counts on the fluid from this compartment. Fluid from the serosal compartment was aspirated, spun down, and resuspended in 100 lambda of tissue culture fluid. Cell counts were attempted using a hemocytometer, and no PMN were present in this compartment $(n=14)$.

Statistical methods. Functional and numerical morphologic data were expressed as means and SEMs for each experimental and control group, respectively. Statistical differences between groups were determined by Student's $t$ test. The relationship between baseline resistances and resistance and chemotactic responses was determined by linear regression.

\section{Results}

Monolayers that were removed from and reinserted into Ussing chambers at intervals of 15-60 min, maintained resistance values similar to their own baseline value (decrease of $7 \pm 4.21 \%$ with reinsertion). Thus, in all experiments, each monolayer could reasonably serve as its own control, and resistance readings were recorded before and after PMN transmigration.

FMLP dose response. Dose-response studies were carried out for $2 \mathrm{~h}$ with varying concentrations of FMLP added to the basal compartment and $10^{7} \mathrm{PMN}$ added to the apical compartment of the monolayers. Maximal resistance and chemotactic responses occurred with gradients of $10^{-6} \mathrm{M}$ to $10^{-7} \mathrm{M}$ FMLP $(96.8 \pm 2.22 \%$ and $81.2 \pm 15.0 \%$ resistance fall, respectively; and $13.2 \pm 2.7 \times 10^{4} / \mathrm{cm}^{2}$ and $13.0 \pm 16.8 \times 10^{4} / \mathrm{cm}^{2}$ PMN chemotactic response, respectively, Fig. 1). Higher or lower concentrations of FMLP diminished both responses. Furthermore, the resistance response correlated well with the chemotactic response ( $r=0.96$, Fig. 2$)$. When data from individual monolayers were analyzed, it became clear that ablation of monolayer resistance (decrease of $>90 \%$ ) occurred when the number of transmigrating PMN reached a density of approximately $10 \times 10^{4} \mathrm{PMN} / \mathrm{cm}^{2}$ (Fig. 3).

$P M N$ dose response. PMN dose-response studies were also carried out by varying the number of PMN applied to the apical surface of the $T_{84}$ monolayers in the presence of a standard FMLP concentration in the basal compartment $\left(10^{-7} \mathrm{M}\right)$. A large resistance response $(83.6 \pm 1.6 \%$ decline) was achieved with $10^{7}$ PMN (Fig. 4). This represented an initial PMN: $\mathrm{T}_{84}$ cell ratio of 10:1 and produced a chemotactic response of $13.4 \pm 1.06 \times 10^{4} \mathrm{PMN} / \mathrm{cm}^{2}$ (Fig. 4). Lower concentrations of PMN lead to lesser resistance and chemotactic responses and $10^{3} \mathrm{PMN}$ in the apical compartment (PMN: $\mathrm{T}_{84}$ ratio of $1: 1,000)$ produced neither resistance nor chemotactic responses (Fig. 4).

Time course. The time courses of the resistance and chemotactic responses were studied by adding $10^{7} \mathrm{PMN}$ to the apical surface of $\mathrm{T}_{84}$ monolayers in the presence of a transepi- 


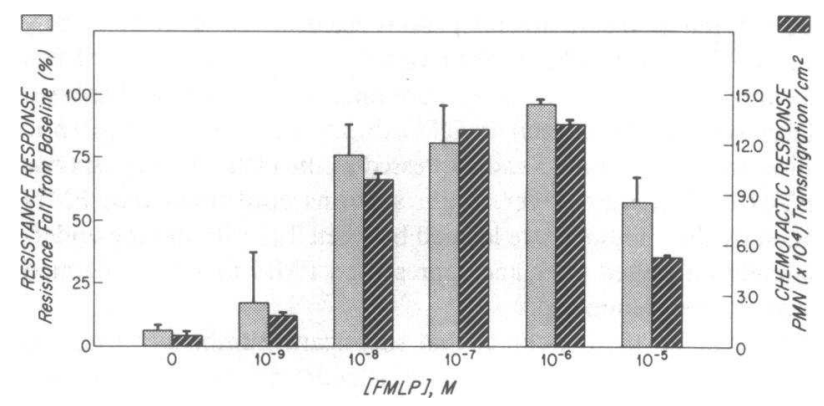

Figure 1. Effects of varying transepithelial gradients of the chemotactic peptide FMLP on resistance and chemotactic responses of $T_{84}$ intestinal cell monolayers. Confluent $T_{84}$ monolayers (7-12 d) on ringmounted Nuclepore filters ( $5 \mu \mathrm{M}$ pore) were elevated on glass beads in plastic tissue-culture wells. Monolayers were oriented such that the apical aspect of the cells faced the upper compartment. $10^{7} \mathrm{PMN}$ were applied to the apical surface and a chemotactic gradient was established across the monolayer with varying doses of FMLP in the basal compartment. Chemotaxis was allowed to progress at $37^{\circ} \mathrm{C}$ in a $95 \%$ air $/ 5 \% \mathrm{CO}_{2}$ atmosphere for $2 \mathrm{~h}$. Resistance response (percent fall in monolayer resistance from its own baseline) and chemotactic response (PMN $\times 10^{4}$ transmigration $\left./ \mathrm{cm}^{2}\right)$ are expressed as $\overline{\mathrm{x}} \pm \mathrm{SEM}$ from three to seven experiments for each bar. Maximal resistance and chemotactic responses occurred with chemotactic gradients of $10^{-7} \mathrm{M}$ and $10^{-6} \mathrm{M}$. We used $10^{-7} \mathrm{M}$ FMLP for subsequent studies.

thelial chemotactic gradient of $10^{-7} \mathrm{M}$ FMLP. Both resistance and chemotactic responses began early. Monolayer resistance fell by $48 \pm 12.6 \%$ by $15 \mathrm{~min}$, reaching a maximum of $83.6 \pm 1.6 \%$ at the end of $1 \mathrm{~h}$ (Fig. 5). Chemotactic responses of transmigrated PMN were $1.7 \pm 0.6 \times 10^{4} / \mathrm{cm}^{2}$ at $15 \mathrm{~min}$, and $13.4 \pm 1.06 \times 10^{4} / \mathrm{cm}^{2}$ at the end of $1 \mathrm{~h}$ (Fig. 5).

Mannitol-inulin flux. To better characterize the size of the epithelial barrier defect produced by transmigration of PMN through $\mathrm{T}_{84}$ monolayers, we performed transepithelial mannitol and inulin flux studies on monolayers incubated for $1 \mathrm{~h}$ with $10^{7} \mathrm{PMN}$ on the apical surface in the presence of a transepithelial chemotactic gradient of $10^{-7}$ M FMLP. Results were compared to those obtained from studies performed on control monolayers. The latter were matched to experimental monolayers for baseline resistance. As shown in Table I, chemotaxis was associated with significant increments in mannitol and inulin flux of approximately threefold greater values than those of controls. The flux rates of mannitol and inulin did not change over the 80 min during which the flux studies were performed indicating that the observed alterations were

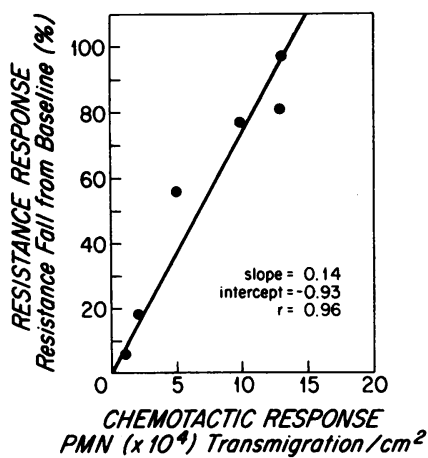

Figure 2. Correlation between resistance response $(\overline{\mathrm{x}} \pm \mathrm{SEM})$ and chemotactic response ( $\overline{\mathrm{x}} \pm$ SEM) for monolayers used in dose response experiments. The resistance response reflects the number of transmigrating PMN. Resistance fall of more than $50 \%$ from baseline correlates with a density of $5 \times 10^{4}$ $\mathrm{PMN} / \mathrm{cm}^{2}$.

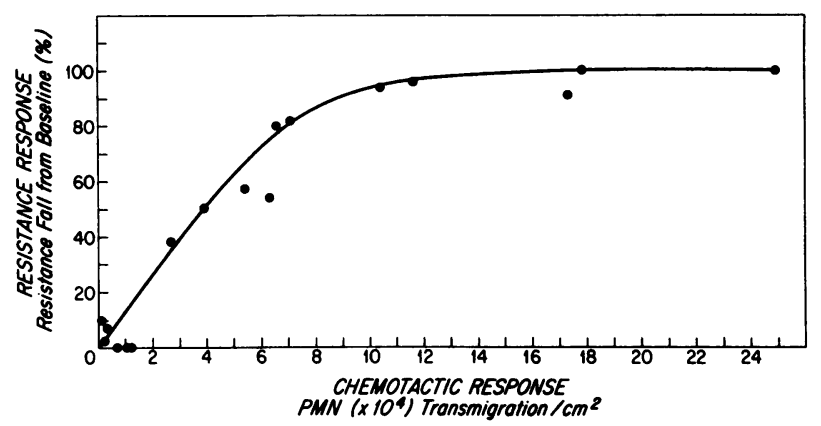

Figure 3. Correlation between resistance response and chemotactic response of individual monolayers. Differing responses were achieved by varying the size of the chemotactic gradient. Progressive increase in monolayer resistance response (i.e., falling resistance) reasonably parallels an increasing density of transmigrating PMN. Monolayer resistance appears to be completely ablated ( $>90 \%$ fall) when densities of migrating PMN reach $\sim 10^{5} \mathrm{PMN} / \mathrm{cm}^{2}$.

not rapidly reversible. To ensure that the increase in permeability of these two extracellular space markers was not due to gross disruption of the monolayers, we performed similar mannitol flux studies on monolayers in which we mechanically disrupted either 10 or $20 \%$ of the surface area (i.e., 90 or $80 \%$ of monolayer was composed of normal epithelium). Such manipulations resulted in enhanced mannitol flux rates exceeding control values by at least 10 -fold (over threefold greater than PMN transmigration induced changes). The effects of PMN transmigration on permeability thus are not attributable to generalized monolayer disruption.

Resealing of $T_{84}$ cell junctions following PMN transmigration. To determine whether $T_{84}$ monolayers could reseal after the PMN-induced resistance drop, we incubated 13 monolayers for 2,4 , or $18 \mathrm{~h}$ in normal media after removal of experimental conditions (i.e., $10^{7} \mathrm{PMN}$ in a FMLP gradient of

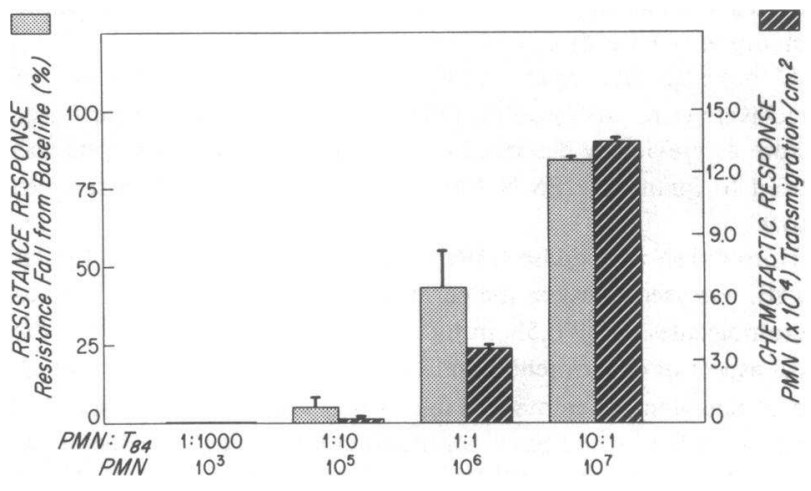

Figure 4. Effect of varying densities of applied PMN on subsequent resistance and chemotactic responses in the presence of a transepithelial chemotactic gradient of $10^{-7}$ M FMLP. PMN suspensions of $10^{3}$ (PMN: $\mathrm{T}_{84}$ ratio of $\left.1: 1000\right) ; 10^{5}$ (PMN: $\mathrm{T}_{84}$ ratio of $\left.1: 10\right) ; 10^{6}$ (PMN: $\mathrm{T}_{84}$ ratio of 1:1); $10^{7}$ (PMN: $\mathrm{T}_{84}$ ratio of 10:1) cells were used. No resistance or chemotactic responses were noted at PMN: $\mathrm{T}_{84}$ cell ratios of 1:1000 and maximal responses occurred at ratios of 10:1. Resistance and chemotactic responses again parallel one another. Resistance and chemotactic responses represent $\bar{x} \pm$ SEM from 3 to 22 experiments. 


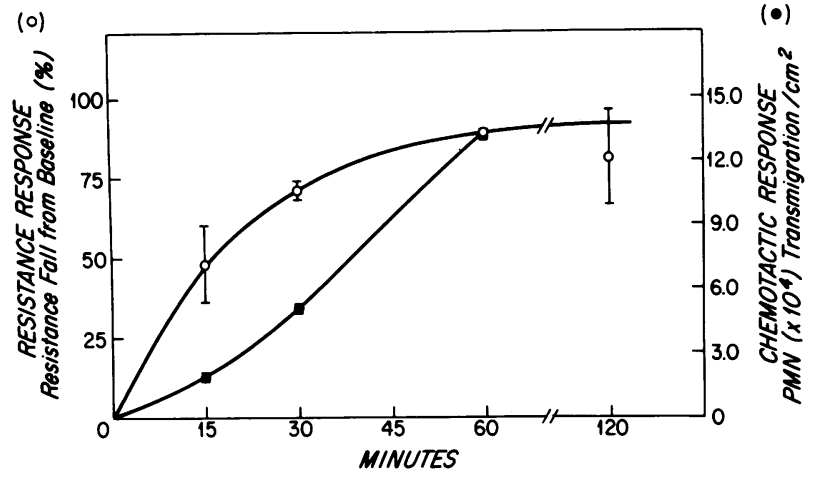

Figure 5. Time course of chemotactic and resistance responses across $\mathrm{T}_{84}$ monolayers. $10^{7} \mathrm{PMN}$ were placed in the presence of a $10^{-7} \mathrm{M}$ gradient of FMLP. Maximal responses were achieved at $60 \mathrm{~min}$. The resistance response occurred rapidly as compared to the chemotactic response. This would be anticipated since resistance is a sensitive index of barrier function that is inordinately affected by the presence of a relatively small subpopulation of enhanced permeability sites (12). Results represent $\bar{x} \pm$ SEM from 5 to 22 experiments.

$10^{-7} \mathrm{M}$ for $30 \mathrm{~min}$ ). As shown in Fig. 6, monolayer resistances recovered to baseline values by $18 \mathrm{~h}$.

PMN transmigration from "serosal" to "mucosal" direction. To determine if PMN transmigration induced effects on epithelial barrier function were independent of the direction of PMN movement, we added $10^{-7}$ M FMLP to the apical compartment of monolayers that had resealed (thus trapping PMN in the subepithelial space) and reincubated them for $1 \mathrm{~h}$. As shown in Fig. 7, a large resistance response was obtained confirming that the effects of PMN transmigration also occur when PMN trapped in the subepithelial spaces of monolayers were allowed to remigrate into the apical compartment (i.e., from serosal to mucosal direction).

Effect of epithelial permeability on PMN transmigration. Others have reasonably proposed that the degree of PMN chemotaxis that occurs across an epithelium would be in part determined by the resistance of that epithelium since gradients of chemotactic agents might be better detected if junctions were more permeable $(7,8)$. To test if the extent of PMN transmigration through $\mathrm{T}_{84}$ monolayers in the presence of a $10^{-7} \mathrm{M}$ FMLP gradient varied with the baseline resistance of the monolayer, we compared resistance and chemotactic responses of individual monolayers against their baseline resistances. For these experiments, monolayers were selected in which baseline resistance ranged from 125 to $2,400 \mathrm{ohm} \mathrm{\textrm {cm } ^ { 2 }}$. In experiments run for 2,1 , or $0.5 \mathrm{~h}\left(10^{7} \mathrm{PMN}\right.$ in a $10^{-7}$ - $\mathrm{M}$ FMLP gradient), the correlation coefficients between baseline

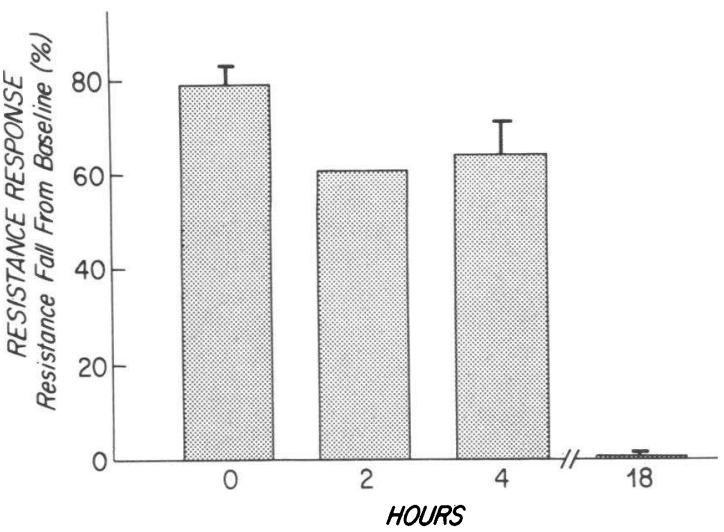

Figure 6. Time course of $\mathrm{T}_{84}$ monolayer resealing after removal of the chemotactic gradient. In these experiments monolayers were exposed to conditions of "maximal" chemotaxis for $30 \mathrm{~min}$ before removing the gradient. Under these conditions resistance responses of $\sim 75 \%$ were obtained and did not change for periods up to $4 \mathrm{~h}$ after gradient removal. However, by $18 \mathrm{~h}$ complete resealing had occurred as evidenced by loss of the resistance response. Resistance response at $0 \mathrm{~h}$ represents that obtained at the end of $30 \mathrm{~min}$ of chemotaxis. Results represent $\bar{x} \pm$ SEM from three experiments for each bar except at $2 \mathrm{~h}$ where $n=2$.

resistances and resistance response were $r=-0.2 ; r=0.16$, and $r=-0.3$, respectively. In experiments run for $1,0.5$, and $0.25 \mathrm{~h}$, the correlation coefficients between baseline resistance and chemotactic response were $r=-0.2, r=-0.3$, and $r$ $=-0.2$. Thus, both resistance response (on a basis of percentage of baseline value) and chemotactic response are independent of the baseline resistance values of any given monolayer.

Effects of chemokinesis on the epithelial barrier. To determine whether the apparent effects of PMN transmigration on monolayer permeability could be due, in part, to FMLP-elicited enhanced random migration (i.e., chemokinesis) of PMN rather than chemotaxis, we incubated six monolayers with both FMLP $10^{-7} \mathrm{M}$ and $10^{7} \mathrm{PMN}$ on the apical surface (i.e., no FMLP gradient) for $1 \mathrm{~h}$ and measured resistance and chemotactic responses occurring under such conditions. The results (Fig. 8) indicate that these conditions produced only trivial differences from controls in both resistance and chemotactic responses. Such results from experiments in which PMN and FMLP were incubated together while overlying $T_{84}$ monolayers also indicate that the resistance response observed during chemotaxis is not due to FMLP-stimulated release of PMN secretory products.

Effect of preexposing monolayers to FMLP. To rule out the possibility that FMLP gradients induced alterations in epithe-

Table I. Effect of PMN Transmigration on Transepithelial Resistance and on Mannitol ( $r=3.6$ A) and Inulin $(r=11-15$ A) Flux

\begin{tabular}{|c|c|c|c|c|}
\hline Monolayers & Baseline R & Postexperiment $\mathbf{R}$ & Postexperiment mannitol & $\begin{array}{l}\text { Postexperiment } \\
\text { inulin }\end{array}$ \\
\hline & $o h m s \mathrm{~cm}^{2}$ & $o h m s \mathrm{~cm}^{2}$ & $f l u x \mu m o l / h \mathrm{~cm}^{2}$ & $f l u x \mu m o l / h \mathrm{~cm}^{2}$ \\
\hline Controls $(n=5)$ & $1,257.4 \pm 359.5$ & $1,238.8 \pm 285.7^{*}$ & $87.6 \times 10^{-4} \pm 36.4^{\ddagger}$ & $5.0 \times 10^{-4} \pm 1.2^{\S}$ \\
\hline Experimental $(n=5)$ & $1,280.8 \pm 321.5$ & $353.4 \pm 154.0$ & $318.8 \times 10^{-4} \pm 132.3$ & $14.3 \times 10^{-4} \pm 5.9$ \\
\hline
\end{tabular}

${ }^{*}$ Control vs. experimental: ${ }^{*} P<0.0005 ;{ }^{\ddagger} P<0.0025 ;{ }^{8} P<0.01$. 


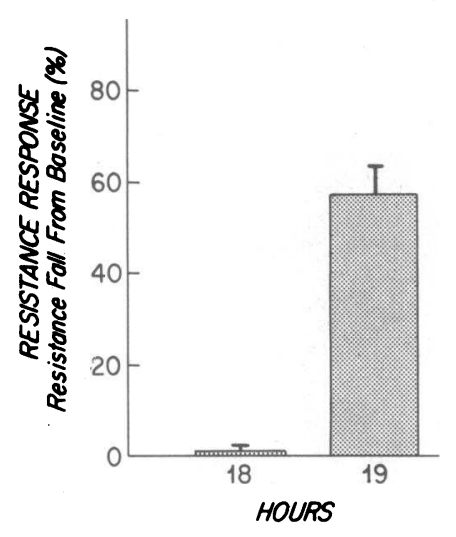

Figure 7. Effects of PMN transmigration from "serosal" to "mucosal" direction. In these experiments, monolayers which had returned to their own baseline resistance values by $18 \mathrm{~h}$ (see Fig. 6) were reincubated for $1 \mathrm{~h}$ with FMLP in the apical compartment. Under these conditions, resistance response of $>50 \%$ was achieved in $1 \mathrm{~h}$.

lial cells that then stimulated PMN to transmigrate, we incubated six monolayers with FMLP $\left(10^{-7} \mathrm{M}\right)$ in the basal compartment with tissue culture fluid in the apical compartment for $1 \mathrm{~h}$. Subsequently, the FMLP was replaced by tissue culture fluid and PMN $10^{7}$ were added to the apical compartment for an additional hour, and resistance and chemotactic responses were measured (Fig. 8). Again, both of these responses were trivial when compared with those obtained under optimal chemotactic gradients.

Morphologic correlates of PMN transmigration. PMN were highlighted by localizing PMN endogenous peroxidases in en face mounted monolayers, thus allowing an overall view of the distribution of transmigrating PMN. 15 min after the onset of the standard experiment $\left(10^{7} \mathrm{PMN}\right.$ in a transepithelial gradient of $10^{-7}$ M FMLP), a speckled pattern of PMN impalement sites was noted (Fig. 9). The density of transmigrated PMN progressively increased with time and by $1 \mathrm{~h} 60-90 \%$ of the monolayer surface was densely stained (Fig. $9 b, c$ ). This speckled pattern of PMN transmigration sites was not observed in separate experiments in which a gradient of FMLP $\left(10^{-7} \mathrm{M}\right)$ was used to drive PMN $\left(10^{7}\right)$ across bare collagencoated filters. The latter displayed a diffusely homogeneous staining pattern. This observation suggests that the distribu-

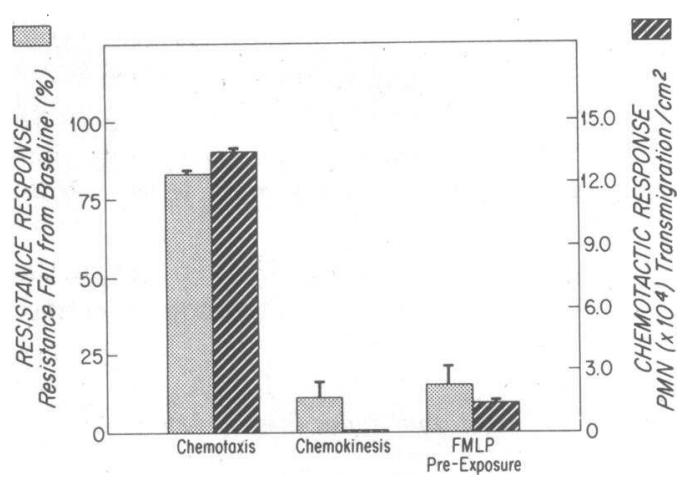

Figure 8. Comparison of resistance and chemotactic response of $T_{84}$ monolayers incubated under: (a) chemotactic conditions $\left(10^{7} \mathrm{PMN}\right.$ apical; $10^{-7}$ M FMLP basal $\times 1 \mathrm{~h} ;(b)$ chemokinetic conditions $\left(10^{7}\right.$ PMN $/ 10^{-7}$ M FMLP combined on apical side $\left.\times 1 \mathrm{~h}\right) ;(c)$ FMLP preexposure $\left(T_{84}\right.$ cells incubated in presence of $10^{-7} \mathrm{M}$ FMLP gradient for $1 \mathrm{~h}$, gradient removed and $10^{7} \mathrm{PMN}$ applied apically for an additional hour). Resistance and "chemotactic" responses are attributable to the presence of a chemotactic gradient.
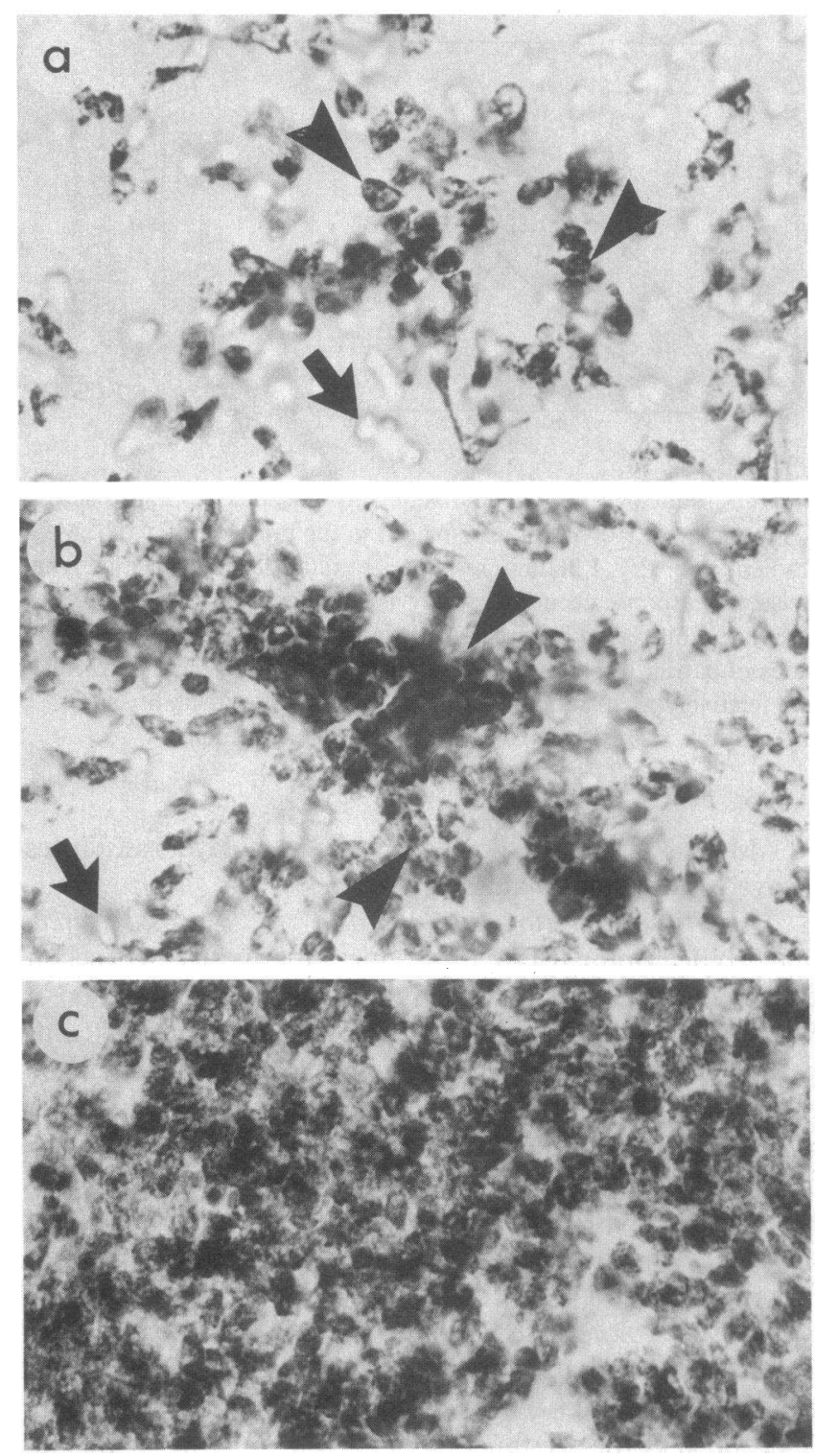

Figure 9. Effect of time on chemotactic response as displayed by light microscopic analysis of monolayers examined en face. $T_{84}$ monolayers stained for peroxidase localization to highlight PMN granules (see Methods). Panel shows speckled pattern of PMN within monolayers ( $10^{7}$ PMN incubated with a $10^{-7} \mathrm{M}$ FMLP gradient); responses at (a) $15 \mathrm{~min}$; (b) $30 \mathrm{~min}$; and (c) $60 \mathrm{~min}$. PMN aggregates become larger and more dense with time until the monolayer is diffusely penetrated by $P M N$ at $60 \mathrm{~min}$. Arrowheads indicate individual PMN while arrows indicate the 5- $\mu \mathrm{M}$ pores in the monolayers $(\times 800)$.

tion of PMN transmigration sites within the epithelium is not entirely random.

$1-\mu \mathrm{m}$ sections of monolayers from standard experiments (15-60 min) showed foci where PMN accumulated both between individual $\mathrm{T}_{84}$ cells and between the monolayer and the underlying collagen-coated filter (Fig. 10). PMN adjacent to the apical surface of the $T_{84}$ cells were rarely seen and PMN below the surface of the collagen table were also rare. Examination of monolayers harvested as early as $15 \mathrm{~min}$ after the FMLP gradient was imposed, also confirmed that PMN association with $\mathrm{T}_{84}$ cell surfaces was an extremely rare or short lived 


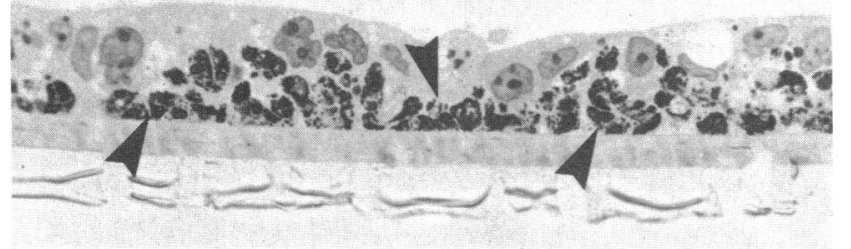

Figure 10. $1 \mu \mathrm{m}$ section of $\mathrm{T}_{84}$ monolayer after PMN transmigration. PMN have migrated into the monolayer $\left(10^{7} \mathrm{PMN} 10^{-7} \mathrm{M}\right.$ FMLP gradient $\times 1 \mathrm{~h}$ ) and can be identified (arrowheads) between $\mathrm{T}_{84}$ cells and beneath $\mathrm{T}_{\mathbf{8 4}}$ cells and the underlying layer of collagen and $\mathrm{Nu}$ clepore filter. The integrity of the monolayer appears intact $(\times 1,280)$.

event even early in the course of the PMN transmigration response. Extensive sampling demonstrated occasional foci in which three to seven PMN appeared tightly apposed to each other and to neighboring $T_{84}$ cells within the zone of the occluding junction. Even more infrequent was the presence of a single PMN migrating through a junctional site. These images of PMN migrating across junctions was present in only $10 \%$ of sections examined ( 15 of 150 sections), even though these sections were obtained from blocks originally identified as harboring areas rich in transmigrating PMN by localization of endogenous peroxidase activity. Given the vast number of PMN that cross the epithelium but the infrequency of capturing an image of PMN crossing the junction, the transjunctional migration event must occur very rapidly (seconds to minutes).

Analysis of thin sections confirmed the presence of clusters of PMN both under the monolayer and within the monolayer in the subjunctional space. When PMN were captured in the process of migrating across the junction (Fig. 11), they appeared to do so by extending thin cytoplasmic pseudopods across the occluding junction and separating adjacent $T_{84}$ cells. When clusters of 4-10 PMN were identified in a transjunctional location (Fig. 12), there was close apposition between cell membranes of $T_{84}$ cells and PMN. Both cell types interdigitated by means of cytoplasmic pseudopodia. Alterations in the cytoskeleton of both $T_{84}$ cells and PMN were present at sites of intimate contact between the plasma membranes of these two cell types. These cytoskeletal alterations consisted chiefly of a dense cortex of intermediate and microfilaments adjacent to the plasma membranes (Fig. 12, inset).

In horseradish peroxidase (HRP) experiments, tracer was applied to the apical surface for the last $20 \mathrm{~min}$ of standard hour-long experiments $\left(10^{7} \mathrm{PMN}\right.$ apical with a FMLP gradient of $10^{-7} \mathrm{M}$ ). The latter allowed us to detect sites of transjunctional permeation since paracellular spaces underlying such sites would be labeled with tracer. In eight experimental monolayers, HRP leaks appeared to be preferentially associated with foci of transmigrating PMN (Fig. $13 \mathrm{~A}$ ). At other sites containing subjunctional PMN, HRP labeled the paracellular spaces but was generally excluded at the level of the occluding junction (Fig. $13 \mathrm{~B}$ ). These findings support the proposal that enhanced permeability is attributable to a subset of junctions that are being actively invaded by PMN. Comparable HRP leaks were generally not present in control monolayers although in one control monolayer paracellular labeling did occur that is unexplained.

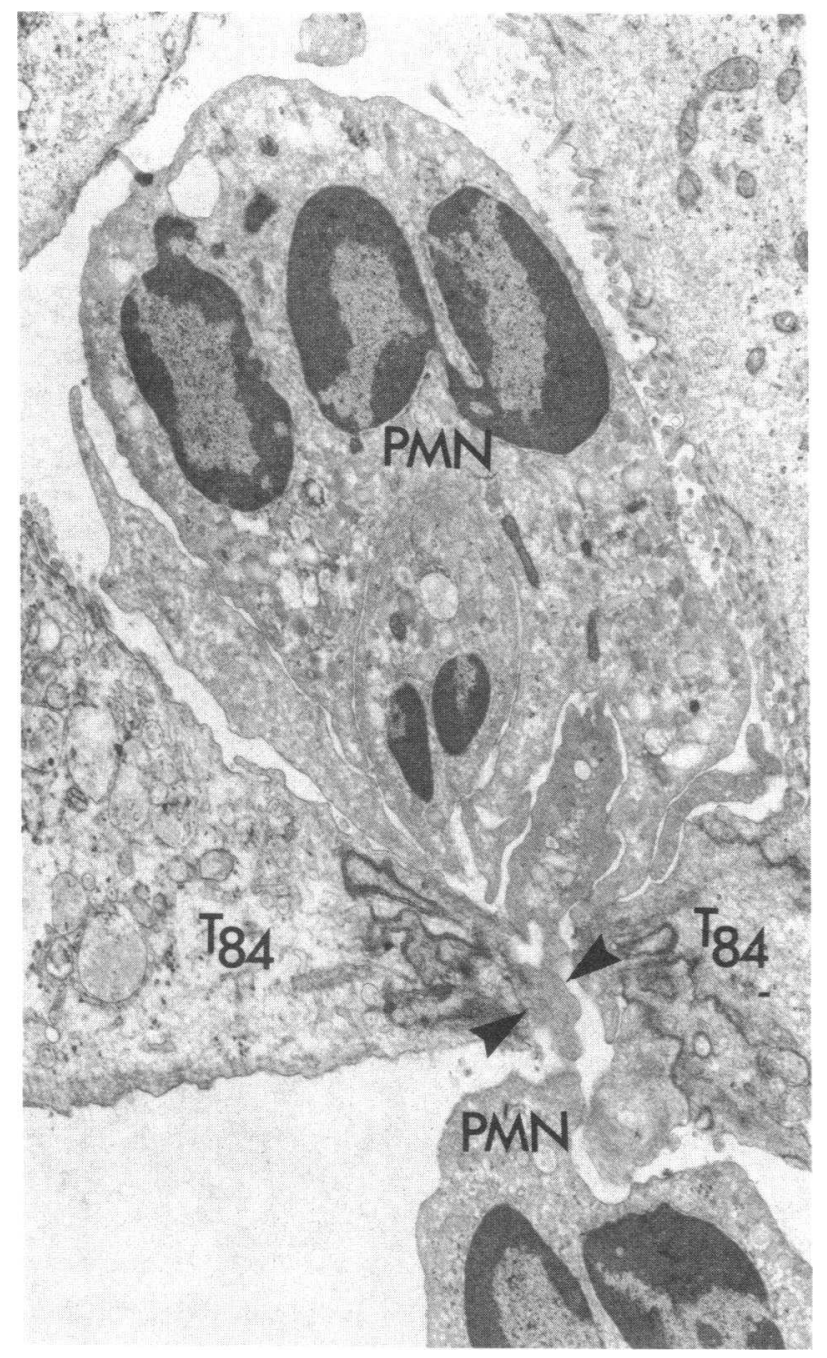

Figure 11. Electron micrograph of PMN indenting the monolayer and passing single file through a junctional impalement site. Transmigration occurs by extension of pseudopodia through the site of epithelial discontinuity (arrowheads). PMN migration is from "mucosal" to "serosal" compartments $(\sim \times 11,000)$.

We also examined $T_{84}$ occluding junction fine structure using the freeze fracture technique. Occluding junction morphology, as assessed by freeze fracture, was indistinguishable between control and experimental monolayers indicating that junctional abnormalities, if present, are highly focal. (We have previously described $T_{84}$ cell tight junction structure in detail [4]).

Effects of PMN transmigration on permeability of monolayers of another cell line. When $10^{7} \mathrm{PMN}$ were placed in the apical compartment and driven across MDCK monolayers with a transepithelial gradient of $10^{-7} \mathrm{M}$ FMLP, mean resistance fall was $67.1 \pm 6.45 \%$. Control MDCK monolayers without a chemotactic gradient showed instability in resistance, in contrast to $\mathrm{T}_{84}$ monolayers, (mean fall in monolayer resistance of $32.1 \pm 9.4 \%$ ) but still had significantly higher resistances than experimental monolayers $(P<0.01)$. Chemotactic responses for experimental MDCK monolayers were $11.1 \pm 0.12 \times 10^{4}$ $\mathrm{PMN} / \mathrm{cm}^{2}$ vs. $0.3 \pm 0.12 \times 10^{4} \mathrm{PMN} / \mathrm{cm}^{2}$ for controls $(P$ $<0.001)$. 


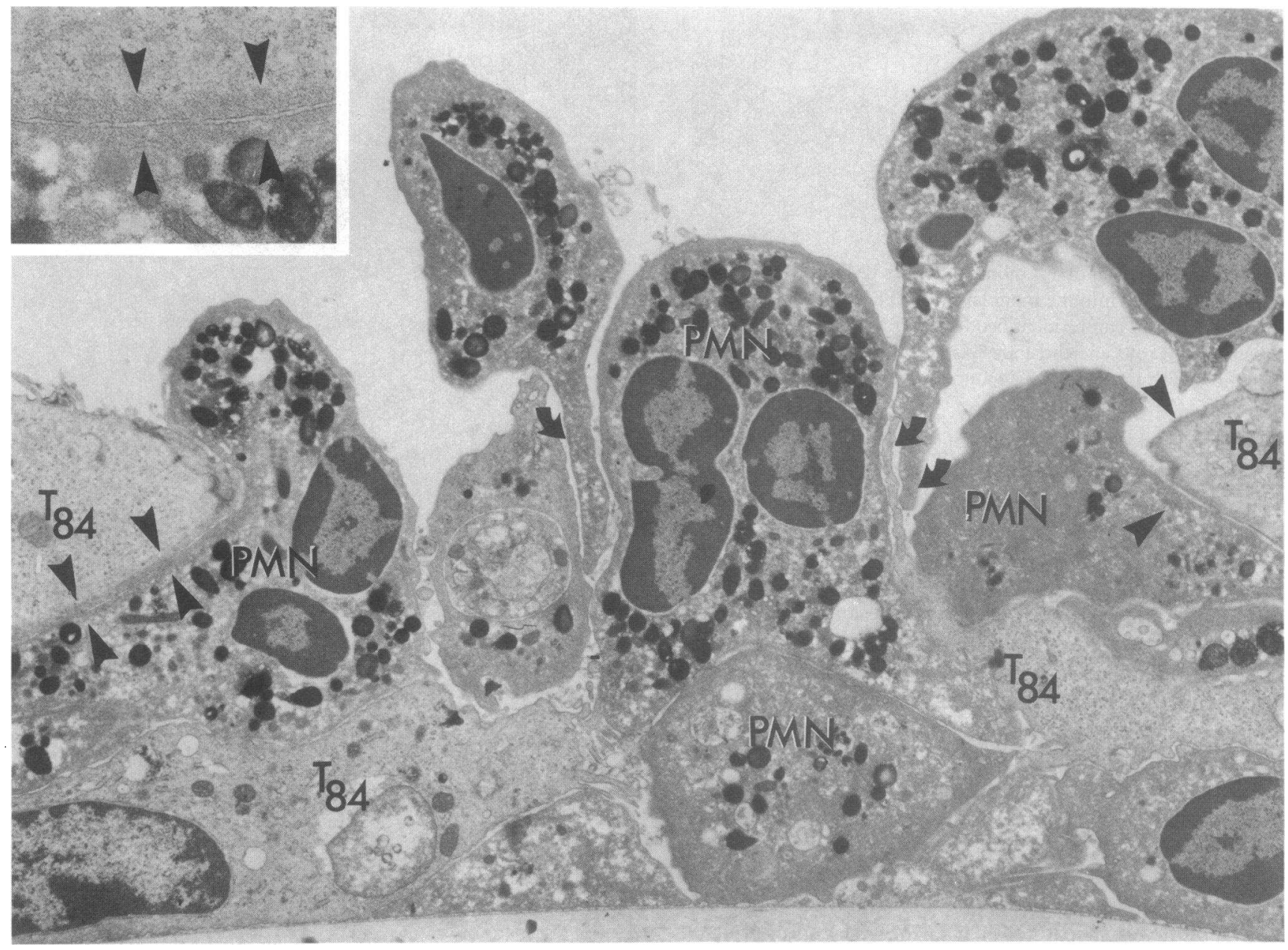

Figure 12. EM depicting an impalement site at which an aggregate of PMN have successfully separated $T_{84}$ cells. PMN pseudopods are intimately associated with each other and with pseudopods of separated $\mathrm{T}_{84}$ cells (arrows). Cytoskeletal rearrangements with the devel-

\section{Discussion}

We show that in response to a chemotactic gradient, human PMN can be induced to migrate across monolayers composed of a human intestinal epithelial cell line ( $\mathrm{T}_{84}$ cells). Due to limitations of experimental design, our data largely are derived from experiments in which transmigration proceeded in a mucosal to serosal direction. However we have been able to reproduce our major resistance findings under conditions of serosal to mucosal transmigration (see Results). The migratory pathway consists of the occluding junction and paracellular space and thus is similar to that taken during PMN migration across natural endothelia and epithelia $(7,8,11)$. A prerequisite of this migratory process is the opening of epithelial intercellular occluding junctions and, indeed, substantial permeability abnormalities, as assessed by resistance, flux, and ultrastructural tracer studies, may occur with PMN transmigration.

In contrast to the major resistance effects which, given the nature of this parameter, might be produced by focal $(4,12)$ permeability abnormalities, measurement of the transjunctional flux of the paracellular tracers mannitol and inulin showed only threefold increases. Given the magnitude of the opment of focal cortical densities in PMN and $\mathrm{T}_{84}$ cells is indicated by arrowheads $(\sim \times 8,500)$ and those on the left side of the EM are better seen in the inset. PMN migration is from mucosal to serosal compartments. $(\sim \times 25,000)$.

epithelial impalement by PMN, it would not have been surprising if fluxes of these markers had been much greater. For instance, mechanical disruption of only $10 \%$ of the epithelial cells in an otherwise unmanipulated monolayer caused mannitol and inulin fluxes to increase 10-fold. Hence it appears that under conditions producing dense PMN transmigration and substantial resistance decreases, junctional permeability defects at any given time may be highly focal. Further evidence for this conclusion include the fact that $(a)$ we were unable to detect structural abnormalities in occluding junctions using freeze fracture techniques; $(b)$ HRP tracer studies demonstrate that although macromolecular permeability defects in the junctional barrier do exist, they are confined to foci with transmigrating PMN; and (c) mannitol and inulin permeabilities are not differentially enhanced in accordance with their differing molecular dimensions.

Such presumptive focal defects in the junctional permeability barrier seen with dense PMN transmigration could result in two ways. Either PMN could migrate through the epithelium at highly selective sites or PMN could diffusely impale the monolayer but rapid resealing might allow detection of only a small subset of impalement sites at any one time. We 


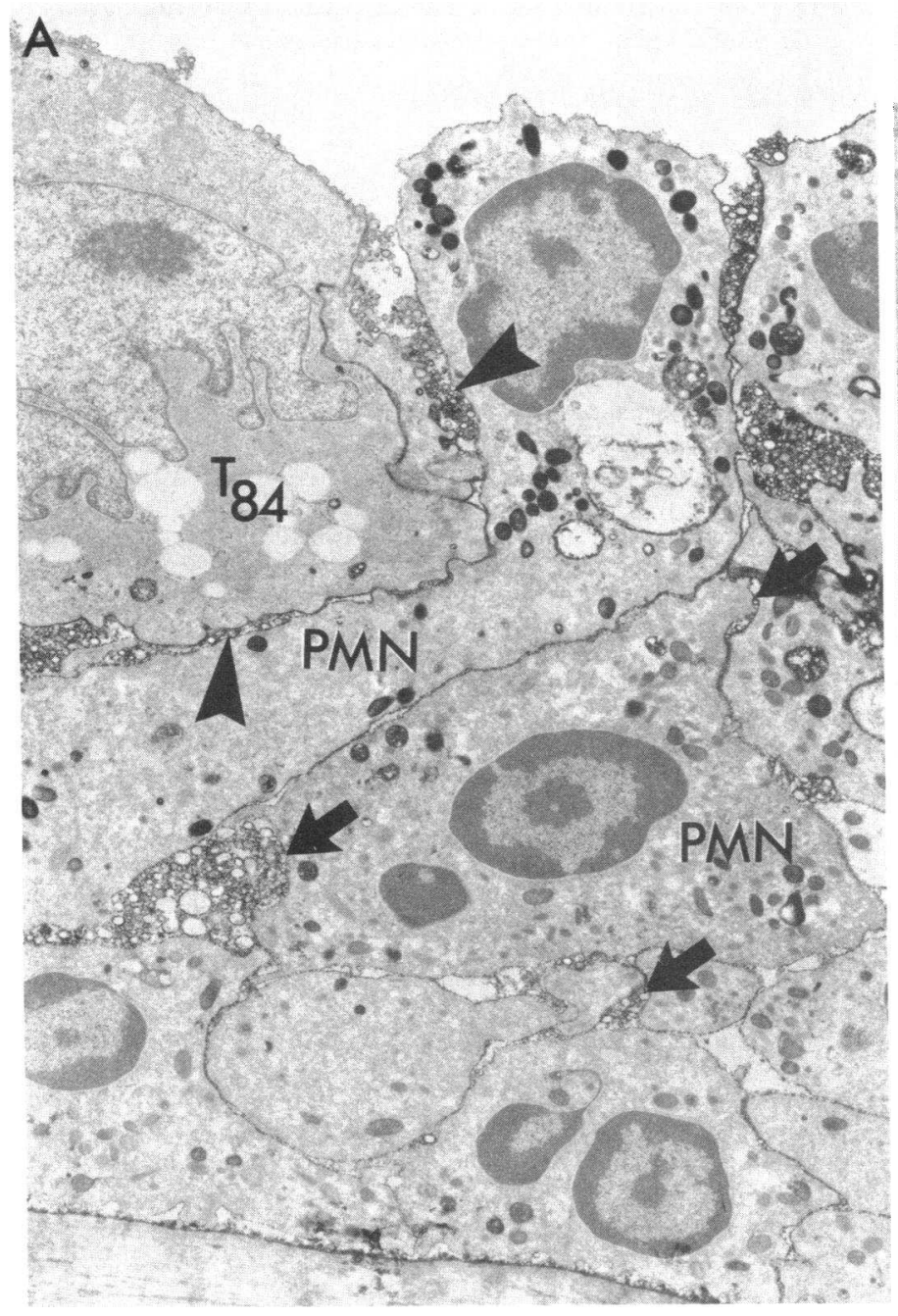

Figure 13. EM derived from monolayers exposed to mucosal HRP during PMN transmigration. $(A)$ At a site of active junctional impalement by PMN, HRP diffusely labels the paracellular spaces (black stain) and is present both between adjacent PMN (arrows) and between PMN and epithelial cells (arrowheads). $(\sim \times 6,000)$. (B) At a

interpret our data as providing evidence that both of these phenomena may be occurring to some extent. In contrast to PMN transmigration across bare, collagen-coated filters, PMN migration across $T_{84}$ monolayers is not entirely random. One speculative but logical basis for this observation is that foci exist in a given monolayer at which the junctional barrier to permeation by FMLP is relatively poor and thus chemotactic gradients are more readily detected.

Our data also indicate that resealing of sites of junctional impalement by PMN does occur. When PMN transmigration is driven under conditions where the initial PMN: $\mathrm{T}_{84}$ cell ratio is 10:1, marked resistance decrements ensue; but if the chemotactic gradient is removed, the monolayers can completely reseal within $18 \mathrm{~h}$. This contrasts with the $5 \mathrm{~d}$ it takes new monolayers plated at confluent density to develop stable resistances $(4,5)$. Evans et al. (13) have also demonstrated that the resistance decrease associated with migration of peritoneal exudate cells across MDCK monolayers can be reversed if conditions that stimulate migration are removed. Similarly, Milks

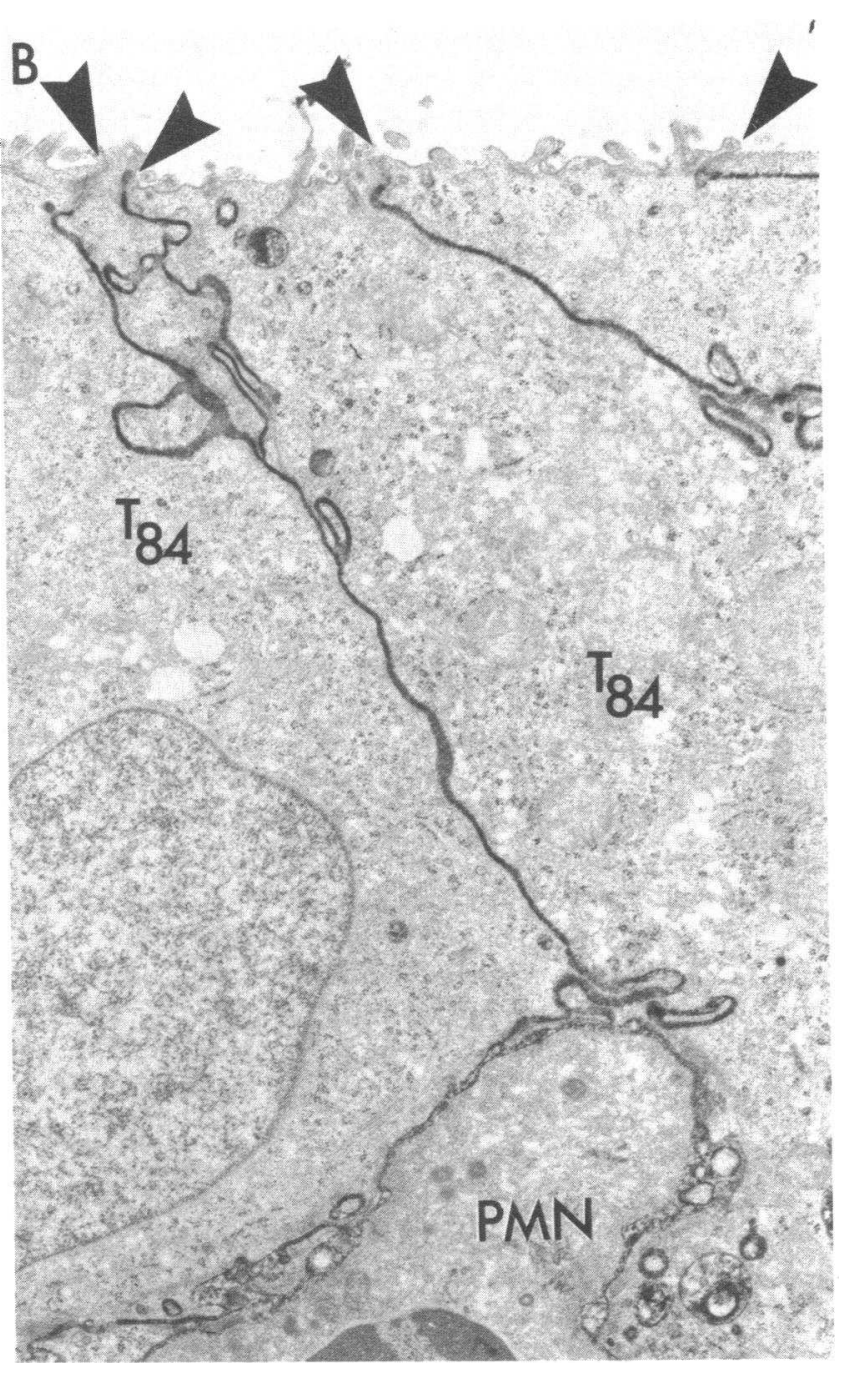

site where PMN transmigration has previously occurred, HRP again diffusely stains the paracellular spaces but appears to be excluded at this time from most of the overlying intercellular occluding junctions (arrowheads). PMN migration is from mucosal to serosal direction. $(\sim \times 5,300)$.

et al. (14) have also recently shown that a renal cell line that forms low resistance monolayers may reseal after PMN transmigration. However, not only can monolayers reseal within hours after a transmigrating "stampede" of PMN, but individual junctions appear to be able to either reseal short-term (seconds-minutes) or not lose their barrier capability at all during more limited degrees of monolayer impalement. This latter conclusion can be derived from circuit model analysis of the resistance response occurring in experiments where the initial $P M N: T_{84}$ ratio was $1: 10$. In these experiments, only trivial resistance decrements were noted although $\sim 10^{3} \mathrm{PMN}$ had crossed the monolayer at widely distributed sites. Knowing the dimensions of the PMN pseudopods that cross the occluding junction (as assessed by electron microscopy of thin sections), the resistance of our Ringer's solution and the depth of the $T_{84}$ occluding junctions (4), we can then calculate the expected resistance response for $10^{3}$ invaded but unsealed junctions (see Appendix). As this analysis shows, $10^{3}$ unsealed transjunctional impalement sites, each having the cross-sectional di- 
mension of a PMN pseudopod, should dramatically reduce monolayer resistance. In striking contrast to this prediction, resistance was observed to decrease from a mean of $1,100 \mathrm{ohm}$ $\mathrm{cm}^{2}$ to $\sim 1,045 \mathrm{ohm} \mathrm{cm}^{2}$. Such data indicate that short-order restitution/maintenance of the junctional barrier to the flow of even ions can be achieved in states in which submaximal transjunctional migration of PMN occurs.

In patients with intestinal inflammatory diseases, PMN epithelial transmigration may be used as a structural marker to judge the severity of disease activity $(1,2)$. Often, but not exclusively, PMN migrate across crypt epithelium and the accumulation of these cells in the crypt lumen represents the classic "crypt abscess" $(1,2)$. Such directional movement of PMN implies the presence of a chemotactic gradient, and indeed chemotactic factors have been identified in the stool of patients with ulcerative colitis (15) and chemotactic peptides, specifically FMLP are known to be synthesized and released by bacteria such as Escherichia coli (16). We have reviewed slides from biopsies and resected specimens of patients with inflammatory bowel disease and counted the number of transmigrated PMN/number of crypt epithelial cells. Such analysis shows that ratios of at least 1:1 are achieved in crypt abscesses. This would correspond to a PMN transmigration density of $10^{6} \mathrm{PMN} / \mathrm{cm}^{2}$ of crypt epithelium and would thus be within the range at which we observe maximal alterations in permeability. We speculate that the increased intestinal permeability described in patients with active inflammatory bowel disease (3) might, at least in part, result from PMN transmigration.

\section{Appendix}

Calculation of the volume $(V)$ of "pores" in the intercellular occluding junctions of our monolayers. (i) This pore volume may be viewed as being equivalent to the volume of Ringer's solution present in the pores and can be calculated using Ohm's law for an electrolytic conductor $R=\mathrm{L} \rho / A$ (17). $R=1,100 \mathrm{ohm} \mathrm{cm} \mathrm{cm}^{2}$ (mean resistance of monolayers used in experiments with $10^{5} \mathrm{PMN}$ ). $L=0.5 \mu \mathrm{M}$ (depth of junctional pores at the level of the intercellular occluding junction (4). $\rho=54 \mathrm{ohm} \mathrm{cm}$ (specific resistivity of our Ringer's solution).

$1.1 \times 10^{3} \mathrm{ohm} \mathrm{cm} \mathrm{cm}^{2}=\frac{\left[5 \times 10^{-5}\right] \mathrm{cm} \times 54 \mathrm{ohm} \mathrm{cm}}{A \mathrm{~cm}^{2}}$

$A \mathrm{~cm}^{2}=\frac{\left[5 \times 10^{-5}\right] \mathrm{cm} \times 54 \mathrm{ohm} \mathrm{cm}}{1.1 \times 10^{3} \mathrm{ohm} \mathrm{\textrm {cm } ^ { 2 }}}$

$=\frac{2.7 \times 10^{-3}}{1.1 \times 10^{3}}$

$A=2.45 \times 10^{-6} \mathrm{~cm}^{2}$

junctional pore volume $=2.45 \times 10^{-6} \mathrm{~cm}^{2} \times\left[5 \times 10^{-5}\right] \mathrm{cm}$

$=2.45 \times 5 \times 10^{-11} \mathrm{~cm}^{3}$

$=122.5 \mu^{3}$

Calculation of volume ( $V$ ) of a PMN pseudopod of diameter $1 \mu \mathrm{M}$ (measured by ultrastructural analysis)

$V=3.14(0.5)^{2} \times 0.5 \mu^{3}$

$=3.14 \times 0.125 \mu^{3}$

$=0.3925 \mu^{3}$

In experiments in which $10^{5} \mathrm{PMN}$ were used, chemotactic response showed 1,000 PMN had crossed the $T_{84}$ cell junctions (Fig. 4). After passage of 1,000 PMN, the junctional pores, if unsealed, have expanded such that the new junctional pore volume $(V)$

$V=122.5 \mu^{3}+\left[.3925 \mu^{3} \times 1,000\right]$

$=122.5 \mu^{3}+392.5 \mu^{3}$

$=515 \mu^{3}$

Area of these pores $\left(A \mathrm{~cm}^{2}\right)=\frac{515 \mu^{3}}{0.5 \mu}$

$=1030 \mu^{2}$

Expected resistance of the monolayer (with unsealed impalement sites) after passage of 1,000 PMN

$R=\frac{\left[5 \times 10^{-5}\right] \mathrm{cm} \times 54 \mathrm{ohm} \mathrm{cm}}{10.3 \times 10^{-6} \mathrm{~cm}^{2}}$

$=\frac{2.7 \times 10^{-3} \mathrm{ohm} \mathrm{cm}}{2}$

$=260 \mathrm{ohm}$

Conclusion. Observed resistance of monolayers used in experiments with $10^{5} \mathrm{PMN}$ after passage of 1,000 PMN shows only a 5\% fall from a baseline of $1,100 \mathrm{ohm} \mathrm{cm}^{2}$ (Fig. 4), whereas by the above calculation it should be $260 \mathrm{ohm}$. Hence, in order to maintain mono-

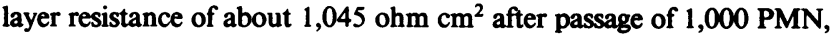
rapid resealing of PMN impalement sites must occur.

\section{Acknowledgments}

The authors thank Dr. Michael Gimbrone for helpful discussions of these data; Ms. Meryl Green and Virginia Hamel for their efforts in the preparation of this manuscript and Ms. Susan Carlson for expert technical support.

This work was supported by National Institutes of Health grant DK-35932 and by a grant from the National Foundation for Ileitis and Colitis. Dr. Nash is supported by U. S. Public Health training grant HL-07066-11. Dr. Madara is supported in part by the American Gastroenterological Association/Ross Research Scholar Award.

\section{References}

1. Kumar, N. B., T. T. Nostrant, and H. D. Appelman. 1982. The histopathologic spectrum of acute self-limited colitis (acute infectioustype colitis). Am. J. Surg. Path. 6:523-529.

2. Yardley, J. H. 1986. Pathology of idiopathic inflammatory bowel disease and relevance of specific cell findings: an overview. In Recent developments in the therapy of inflammatory bowel disease. Proceedings of a symposium. Myerhoff Center for Digestive Disease at Johns Hopkins, Baltimore, MD. 3-9.

3. Hawker, P. C., J. S. McKay, and L. A. Turnberg. 1980. Electrolyte transport across colonic mucosa from patients with inflammatory bowel disease. Gastroenterology. 79:508-511.

4. Madara, J. L., and K. Dharmsathaphorn. 1985. Occluding junction structure-function relationships in a cultured epithelial monolayer. J. Cell Biol. 101:2124-2133.

5. Madara, J. L., J. Stafford, K. Dharmsathaphorn, and S. Carlson. 1987. Structural analysis of a human intestinal epithelial cell line. Gastroenterology. 92:1133-1145.

6. Dharmsathaphorn, K., J. A. McRoberts, K. G. Mandel, L. D. Tisdale, and H. Masui. 1984. A human colonic tumor cell line that maintains vectorial electrolyte transport. Am. J. Physiol. 246(Gastrointest. Liver Physiol. 9):G204-G208.

7. Cramer, E. B., L. C. Milks, and G. K. Ojakian. 1980. Transepi- 
thelial migration of human neutrophils: an in vitro model system. Proc. Natl. Acad. Sci. USA. 77:4069-4073.

8. Milks, L. C., M. J. Brontoli, and E. B. Cramer. 1983. Epithelial permeability and the transepithelial migration of human neutrophils. J. Cell Biol. 96:1241-1247.

9. Bevilacqua, M. P., J. S. Pober, M. E. Wheeler, R. S. Cotran, and M. A. Gimbrone, Jr. 1985. Interleukin I acts on cultured human vascular endothelium to increase the adhesion of polymorphonuclear leukocytes, monocytes and related leukocyte cell lines. J. Clin. Invest. 76:2003-2011.

10. Graham, R. C., and M. J. Karnovsky. 1966. The early stages of absorption of injected horseradish peroxidase in the proximal tubules of mouse kidney: Ultrastructural chemistry by a new technique. $J$. Histochem. Cytochem. 14:291-300.

11. Marchesi, V. T. 1961. The site of leukocyte emigration during inflammation. Q. J. Exp. Physiol. 46:115-118.

12. Marcial, M. A., S. L. Carlson, and J. L. Madara. 1984. Partitioning of paracellular conductance along the ileal crypt-villus axis. A hypothesis based on structural analysis with detailed consideration of tight junction structure-function relationships. J. Membr. Biol. 80:5970.

13. Evans, C. W., J. E. Taylor, J. D. Walker, and N. L. Simmons. 1983. Transepithelial chemotaxis of rat peritoneal exudate cells. Br. $J$. Exp. Pathol. 64:644-654.

14. Milks, L. C., G. P. Conyers, and E. B. Cramer. 1986. The effect of neutrophil migration on epithelial permeability. J. Cell Biol. 103:2729-2738.

15. Franklin, M. L., R. Cisneros, and A. B. Onderdonk. 1980. Chemotactic factors in the stool of patients with ulcerative colitis. Am. J. Clin. Nutr. 33:2540. (Abstr.)

16. Marasco, W. A., S. H. Phan, H. Krutzsch, H. J. Showell, D. E. Feltner, R. Nairn, E. L. Becker, and P. A. Ward. 1984. Purification and identification of formyl-methionyl-leucyl-phenylalanine as the major peptide neutrophil chemotactic factor produced by Escherichia coli. $J$. Biol. Chem. 259:5430-5439.

17. Woodbury, J. W. 1965. Action potential: properties of excitable membranes. In Physiology and Biophysics. T. C. Ruch and H. D. Patton, editors. W. B. Saunders Co., Philadelphia and London. 61-72. 